\title{
SPHERICAL FUNCTIONS ON A p-ADIC CHEVALLEY GROUP
}

\author{
BY I. G. MACDONALD
}

Communicated by G. W. Mackey, November 27, 1967

1. Introduction. In [6] Satake set up a general theory of zonal spherical functions on a reductive linear algebraic group over a $p$-adic field. One problem left open was the determination of the explicit form of the spherical functions and the Plancherel measure. In this note we shall present explicit formulas for these things in the case of a Chevalley group. Detailed proofs will appear elsewhere.

2. Notation. Let $\mathrm{o}$ be a complete discrete valuation ring and $K$ its field of fractions. Choose a generator $\pi$ of the maximal ideal of $\mathfrak{D}$. Suppose that the residue field of $\boldsymbol{D}$ is finite and has $q$ elements. Then $K$ is locally compact (and totally disconnected) with respect to the valuation topology.

Let $\mathfrak{g}$ be a complex semi-simple Lie algebra, $\mathfrak{h}$ a Cartan sub-algebra of $\mathfrak{g}$, and $V$ the vector-space dual of $\mathfrak{h}$. The dual $V^{*}$ of $V$ is then identified with $\mathfrak{h}$. If $\xi \in \mathfrak{h}=V^{*}$ and $\eta \in V$ we write $(\xi, \eta)$ for the value of $\eta$ at $\xi$ (or of $\xi$ at $\eta$ ). Let $\Delta C V$ be the set of (nonzero) roots of $g$ relative to $\mathfrak{h}$, and for each root $\alpha$ let $\alpha^{*} \in V^{*}$ be the corresponding coweight. Choose a system of fundamental roots $\Pi$ in $\Delta$; we can then speak of positive and negative roots. The roots generate a free abelian group $R \subset V$ of $\operatorname{rank} l=\operatorname{dim} V$, and $\Pi$ is a basis of $R$. Let $R^{*}$ denote the set of all $\xi \in V^{*}$ such that $(\xi, \alpha) \in \boldsymbol{Z}$ for all roots $\alpha$. Then $R^{*} \cong \operatorname{Hom}(R, Z)$ and is a free abelian group of rank $l$ contained in $V^{*}$, and contains all the coweights $\alpha^{*}$. Let $R_{+}^{*}$ be the set of all $\lambda \in R^{*}$ such that $(\lambda, \alpha) \geqq 0$ for all positive roots $\alpha$.

The Weyl group $W$ of $\mathfrak{g}$ (relative to $\mathfrak{h}$ ) acts on $\mathfrak{h}$ and hence on $V$ by transposition. Any element of $R^{*}$ can be brought into $R_{+}^{*}$ by the action of some element of $W$. For each $w \in W$ let $n(w)$ denote the number of positive roots $\alpha$ such that $w(\alpha)<0$, and let $P(t)$ denote the Poincare polynomial

$$
P(t)=\sum t^{n(w)}
$$

summed over all $w \in W$. More generally, if $\lambda \in R^{*}$ let

$$
P_{\lambda}(t)=\sum t^{n(w)}
$$

summed over all $w \in W$ such that $w(\lambda)=\lambda$. Then $P_{\lambda}(t)$ divides $P(t)$, and we put 


$$
P^{\lambda}(t)=P(t) / P_{\lambda}(t) .
$$

Let $X_{\alpha}(\alpha \in \Delta) ; \alpha^{*}(\alpha \in \Pi)$ be a Chevalley basis of $\mathfrak{g}$ relative to $\mathfrak{h}$. Let $g_{z}$ be the Lie sub-ring of $g$ generated by these basis elements, and let $\mathfrak{g}_{K}=K \otimes \mathfrak{g}_{z}$ and $\mathfrak{g}_{0}=\mathfrak{D} \otimes \mathfrak{g}_{z}$. For each $t \in K$ and $\alpha \in \Delta$ let $x_{\alpha}(t)$ $=\exp \left(t\right.$ ad $\left.X_{\alpha}\right)$, which is an automorphism of $\mathfrak{g}_{K}$. For each homomorphism $\chi: R \rightarrow K^{*}$ let $h(\chi)$ be the automorphism of $g_{K}$ which leaves each basis element $\alpha^{*}$ fixed and maps $X_{\alpha}$ to $\chi(\alpha) X_{\alpha}$ for all $\alpha \in \Delta$. Let $G$ be the group of automorphisms of $g_{K}$ generated by the $x_{\alpha}(t)$ and the $h(x)$.

We define sub-groups $U, H, N$ of $G$ as follows. $H$ is generated by the $h(\chi)$ and is isomorphic to $\operatorname{Hom}\left(R, K^{*}\right) ; N$ is generated by the $x_{\alpha}(t)$ for all $t \in K$ and all positive roots $\alpha$; and $U$ is the stabilizer in $G$ of the lattice go. The group $G$ is locally compact and $U$ is a maximal compact sub-group of $G$, and is open in $G$.

The choice of generator $\pi$ enables us to embed $Z$ in $K^{*}$ by $n \mapsto \pi^{n}$, and hence $R^{*} \cong \operatorname{Hom}(R, Z)$ in $H \cong \operatorname{Hom}\left(R, K^{*}\right)$. If $\lambda \in R^{*}$, let $\pi^{\lambda}$ denote the image of $\lambda$ in $H$; explicitly, $\pi^{\lambda}$ maps each $X_{\alpha}$ to $\pi^{(\lambda, \alpha)} X_{\alpha}$. Finally, let $H^{0}$ (resp. $H_{+}^{0}$ ) be the image of $R^{*}$ (resp. $R_{+}^{*}$ ) in $H$. Then [1], [4]:

(2.4) Cartan decomposition. $G=U H_{+}^{0} U$, and $G$ is the disjoint union of the double cosets $U \pi^{\lambda} U$ where $\lambda \in R_{+}^{*}$.

(2.5) Irwasawa decomposition. $G=U H^{0} N$, and $G$ is the disjoint union of the double cosets $U \pi^{\lambda} N$ where $\lambda \in R^{*}$.

3. Spherical functions. In this paragraph $G$ is any unimodular separable locally compact topological group, and $U$ is a compact sub-group of $G$. Let $L(G, U)$ be the $C$-vector space of all complexvalued continuous functions on $G$ which have compact support and are bi-invariant with respect to $U$, that is to say are constant on each double coset $U x U$ of $U$ in $G$. Fix a Haar measure $d x$ on $G$ and define multiplication in $L(G, U)$ to be convolution:

$$
(f * g)(x)=\int_{G} f\left(x y^{-1}\right) g(y) d y .
$$

Then $L(G, U)$ is a $C$-algebra. Assume now that $L(G, U)$ is commutative. Then a zonal spherical function (z.s.f. for short) on $G$ relative to $U$ is defined to be a complex-valued continuous function $\omega$ on $G$ which satisfies the following three conditions: (1) $\omega$ is bi-invariant with respect to $U$; (2) $\omega(1)=1$; (3) $f * \omega=\lambda_{f} \omega$ for all $f \in L(G, U)$, where $\lambda_{f}$ is a complex number depending on $f$. For the theory of spherical functions we refer to [2], [3], [7]. 
A z.s.f. $\omega$ is said to be of positive type if

$$
\int_{G} \int_{G} \omega\left(x y^{-1}\right) \phi(x) \overline{\phi(y)} d x d y \geqq 0
$$

for all continuous functions $\phi: G \rightarrow C$ with compact support. Let $\Omega$ (resp. $\Omega^{+}$) denote the set of all z.s.f. (resp. z.s.f. of positive type) on $G$ relative to $U$. For each $f \in L(G, U)$ the Fourier transform of $f$ is the function on $\Omega$ defined by

$$
\hat{f}(\omega)=\int_{G} f(x) \omega\left(x^{-1}\right) d x .
$$

The basic fact [2] is that there exists a unique positive measure $d \omega$ on $\Omega^{+}$, called the Plancherel measure, such that

$$
\int_{\theta}|f(x)|^{2} d x=\int_{\Omega+}|\hat{f}(\omega)|^{2} d \omega
$$

for all $f \in L(G, U)$.

4. Spherical functions on a Chevalley group. Now let $G$ and $U$ be as defined in $\$ 2$. An elementary argument, based on the existence of an automorphism of $\mathfrak{g}$ which sends every coweight $\alpha^{*}$ to its negative, shows that $L(G, U)$ is commutative (see [3, p. 408] for the corresponding result in the real case).

The following construction gives z.s.f.'s on $G$ (relative to $U$ ). Let $\phi: H^{0} \rightarrow C^{*}$ be a homomorphism, and use the Iwasawa decomposition (2.5) to extend $\phi$ to a function on the whole of $G$ : if $x \in U h N$ with $h \in H^{0}$, define $\phi(x)$ to be $\phi(h)$. Now let

$$
\omega_{\phi}(x)=\int_{0} \phi\left(x^{-1} u\right) d u
$$

where $d u$ is Haar measure on the compact group $U$, normalized so that the measure of $U$ is 1 . Then $\omega_{\phi}$ is a z.s.f. on $G$ relative to $U$, and moreover all z.s.f. are obtained in this way [6].

It is convenient to parametrize the $\omega_{\phi}$ as follows [6]. Given $\phi: H^{0} \rightarrow C^{*}$, there exists $s_{0} \in V$ such that $\phi\left(\pi^{\lambda}\right)=q^{-\left(\lambda, 0_{0}\right)}$ for all $\lambda \in R^{*}$; this $s_{0}$ is not uniquely determined by $\phi$, but is unique modulo $(2 \pi i / \log q) R$. Let

$$
\sigma=\frac{1}{2} \text { (sum of positive roots) }
$$

and let $s=s_{0}+\sigma$. Then we take $s \in V$ as parameter for the z.s.f. $\omega_{\phi}$, and we write $\omega_{\diamond}$ in place of $\omega_{\phi}$. The advantage of this parametrization 
is that $\omega_{s}=\omega_{s^{\prime}}$ if and only if $s^{\prime} \equiv w s(\bmod (2 \pi i / \log q) R)$ for some $w \in W$. The integral (4.1) now becomes

$$
\omega_{s}(x)=\int_{U} q^{(\mu(x-1 u), \sigma-s)} d u
$$

where $\mu$ is the projection of $G$ on $R^{*}=U \backslash G / N$.

Since $\omega_{s}$ is bi-invariant with respect to $U$, it follows from (2.4) that $\omega_{s}$ is uniquely determined by its values on $H_{+}^{0}$.

For each $s \in V$ which does not lie on a root hyperplane, i.e. which is such that $\left(\alpha^{*}, s\right) \not \equiv 0(\bmod (2 \pi i / \log q))$ for all $\alpha \in \Delta$, let

$$
c(s)=\prod_{\alpha>0} \frac{1-q^{-1-\left(\alpha^{*}, s\right)}}{1-q^{-\left(\alpha^{*}, 8\right)}} .
$$

Then we have the following formula.

Theorem 1. Let $\lambda \in R_{+}^{*}$ and let $s \in V$ be as above. Then

$$
\omega_{s}\left(\pi^{\lambda}\right)=\frac{1}{P\left(q^{-1}\right)} \sum_{w \in W} q^{(\lambda, w s-\sigma)} c(w s)
$$

where the polynomial $P$ is that defined in (2.1).

This is proved by computing the integral (4.3). For this purpose the Iwahori decomposition of $U[4]$ is used to split up $U$ into a disjoint union of double cosets $U_{w}(w \in W)$, and the integral over $U_{w}$ is calculated by induction on the integer $n(w)$. (It is not the case that the terms in the sum on the right-hand side of (4.5) correspond to the integrals over the double cosets $U_{w}$; it is more complicated than that.)

Formula (4.5) is still valid, suitably interpreted, if $s$ lies on a root hyperplane in $V$. Let $A=Q\left[R^{*}\right]$ be the rational group algebra of $R^{*}$ and let $e^{\lambda}$ denote the canonical image of $\lambda \in R^{*}$ in $A$. Consider the fraction

$$
Q_{\lambda}=\sum_{w \in W} e^{-w \lambda} \prod_{\alpha>0} \frac{1-q^{-1} e^{w \alpha^{*}}}{1-e^{w \alpha^{*}}}
$$

where $\lambda \in R^{*}$. On the face of it, $Q_{\lambda}$ is an element of the field of fractions of the integral domain $A$. In fact it is easy to see that $Q_{\lambda} \in A$. Now any homomorphism $R^{*} \rightarrow C^{*}$ extends to a ring homomorphism $A \rightarrow \mathrm{C}$; if $\psi_{s}: A \rightarrow \mathrm{C}$ is the extension of $\lambda_{1} \mapsto q^{-(s, \lambda)}$, then (4.5) takes the form 


$$
\omega_{\bullet}\left(\pi^{\lambda}\right)=\frac{q^{-(\lambda, \sigma)}}{P\left(q^{-1}\right)} \psi_{\bullet}\left(Q_{\lambda}\right)
$$

and is valid for all $s \in V$.

Theorem 1 can be reformulated as a purely algebraic statement about the structure of the ring $L(G, U)$. For each $\lambda \in R^{*}$, let $\chi_{\lambda}$ denote the characteristic function of the double coset $U \pi^{\lambda} U$ in $G$. The $\chi_{\lambda}$ with $\lambda \in R_{+}^{*}$ form a basis of $L(G, U)$ as a $C$-vector space. Their Fourier transforms $\hat{\chi}_{\lambda}$ are functions on the space $\Omega$ of z.s.f. and hence can be regarded as $W$-invariant functions on $V$. In fact $[6, \S 6]$ they are polynomial functions and hence may be regarded as elements of $Q\left[R^{*}\right]$, or of $C\left[R^{*}\right]$. Thus Fourier transformation gives rise to a homomorphism $L(G, U) \rightarrow C\left[R^{*}\right]$, and Theorem 1 then takes the following form:

THEOREM $1^{\prime}$. Let $C\left[R^{*}\right]^{W}$ be the sub-algebra of $W$-invariant elements of the group algebra $C\left[R^{*}\right]$, and let $f: L(G, U) \rightarrow C\left[R^{*}\right]^{\text {W }}$ be the C-linear mapping defined by

$$
f\left(\chi_{\lambda}\right)=\frac{q^{\lambda, \sigma)}}{P^{\lambda}\left(q^{-1}\right)} Q_{\lambda},
$$

where $Q_{\lambda}$ is defined in (4.6), $\sigma$ in (4.2) and $P^{\lambda}$ in (2.3). Then $f$ is a C-algebra isomorphism.

5. Plancherel measure. Let $\Omega^{+}$be the space of z.s.f. of positive type on $G$ relative to $U$. Let $V_{0}$ be the real vector subspace of $V$ spanned by $\Delta$, so that $V$ is the complexification of $V_{0}$. If $s \in i V_{0}$, then $\omega_{s}$ is a z.s.f. of positive type. The $\omega_{s}$ with $s \in i V_{0}$ form a subspace $\Omega_{0}^{+}$of $\Omega^{+}$; in general $\Omega_{0}^{+}$is not the whole of $\Omega^{+}$(see [6, Appendix 2], for the case of $P G L(2, K)$ ), but we can afford to ignore the rest of $\Omega^{+}$because it will have Plancherel measure zero. The space $\Omega_{0}^{+}$is the quotient of $i V_{0}$ firstly by the lattice $(2 \pi i / \log q) R$ and then by the Weyl group $W$, i.e. it is of the form $T / W$ where $T$ is an $l$-dimensional real torus. Let $d s$ be a Euclidean measure on $i V_{0}$ and let $d x$ be a Haar measure on $G$. Then

TheOREM 2. The Plancherel measure dw on $\Omega^{+}$is concentrated on $\Omega_{0}^{+}$. If $d s$ and $d x$ are suitably normalized, then

$$
d \omega_{0}=|c(s)|^{-2} d s \quad\left(s \in i V_{0}\right)
$$

where $c(s)$ is defined in (4.4), so that

$$
|c(s)|^{-2}=\prod_{\alpha} \frac{1-q^{-\left(\alpha^{*}, s\right)}}{1-q^{-1-\left(\alpha^{*}, 0\right)}}
$$


the product being taken over all roots $\alpha$, positive and negative.

This is an easy consequence of Theorem 1. It agrees with Mautner's result [5] for the case of $\operatorname{PGL}(2, K)$.

\section{REFERENCES}

1. F. Bruhat, Sur une classe de sous-groupes compacts maximaux des groupes de Chevalley sur un corps p-adique, Inst. Hautes Etudes Sci. Publ. Math. 23 (1964), 46-74.

2. R. Godement, Introduction aux travaux de A. Selberg, Sém. Bourbaki 9 (19561957).

3. S. Helgason, Differential geometry and symmetric spaces, Academic Press, New York, 1962.

4. $\mathrm{N}$. Iwahori and $\mathrm{H}$. Matsumoto, On some Bruhat decomposition and the structure of the Hecke rings of $\mathfrak{p}$-adic Chevalley groups, Inst. Hautes Etudes Sci. Publ. Math. 25 (1965), 5-48.

5. F. I. Mautner, Spherical functions over p-adic fields, Amer. J. Math. 80 (1958), 441-457.

6. I. Satake, Theory of spherical functions on reductive algebraic groups over $p$-adic fields, Inst. Hautes Etudes Sci. Publ. Math. 18 (1963), 5-69.

7. T. Tamagawa, On Selberg's trace-formula. J. Fac. Sci. Univ. Tokyo 8 (1960), 363-386.

OxFord UNIVERSITY, ENGLAND 